\title{
Semi-Interpenetrating Polymer Networks involving Agro-Based Materials
}

H. N. Cheng ${ }^{1, *}$ and Atanu Biswas ${ }^{2}$

${ }^{1}$ Southern Regional Research Center, USDA Agricultural Research Service, New Orleans, LA, United States

${ }^{2}$ National Center for Agricultural Utilization Research, USDA Agricultural Research Service, Peoria, IL 61604, United States.

At USDA we are interested in the conversion of agro-based materials to value-added products. One approach we have found to be particularly versatile is the design and synthesis of semiinterpenetrating polymer networks (SIPN's) that permit a range of new materials to be made comprising biobased materials and synthetic polymers. Thus, a monomeric system (M1) can be mixed with a biobased material (M2), and when polymerization occurs, the resulting product is a SIPN containing the resulting M1 polymer and the M2 biobased material. As an example, a facile polymerization that can be used for this purpose is polyurethane formation. We have made several polyurethanes from simple sugars and selected carbohydrates (e.g., sucrose, sorbitol, cyclodextrin, maltodextrin, and xylan) through the reaction with a diisocyanate. The weight ratio of the diisocyanate versus the carbohydrate needs to be customized in each case in order to optimize the molecular weight and thermal properties required for a given application. Some of these polyurethanes (M1's) can then be used to form SIPN's with biobased materials (M2's) such as poly(lactic acid) and soybean oil. Other polymers [e.g., polycaprolactone and poly(vinyl pyrrolidone)] can be incorporated as M2 into the polyurethane in the same way. The final properties of the SIPN reflect both the properties of the M1 polymer and the M2 material. Variations of this theme can be used to produce SIPN's involving other biobased materials and different types of polymerization.

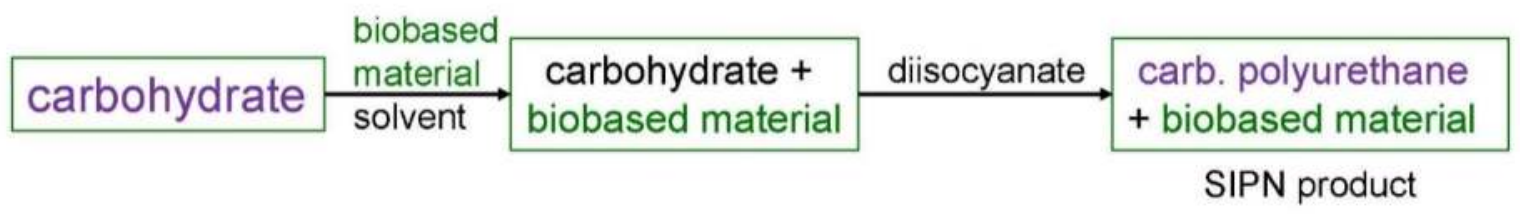

